\title{
Effects of the SARS-CoV-2 global pandemic on U.S. rheumatology outpatient care delivery and use of telemedicine: an analysis of data from the RISE registry
}

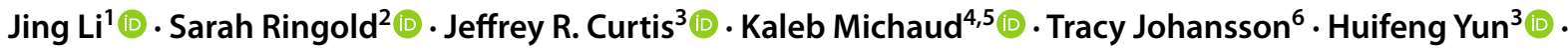 \\ Jinoos Yazdany ${ }^{1}$ (1) $\cdot$ Gabriela Schmajuk ${ }^{1,7}$ (1)
}

Received: 10 June 2021 / Accepted: 20 July 2021 / Published online: 2 August 2021

(c) The Author(s), under exclusive licence to Springer-Verlag GmbH Germany, part of Springer Nature 2021

\begin{abstract}
The SARS-CoV-2 global pandemic resulted in major disruptions to medical care. We aimed to understand changes in outpatient care delivery and use of telemedicine in U.S. rheumatology practices during this period. Rheumatology Informatics System Effectiveness (RISE) is a national, EHR-enabled registry that passively collects data on all patients seen by participating practices. Included practices were required to have been participating in RISE from January 2019 through August $2020(N=213)$. We compared total visit counts and telemedicine visits during March-August 2020 to March-August 2019 and stratified by locations in states with shelter-in-place (SIP) orders. We assessed characteristics of patients within each practice, including primary rheumatic diagnosis and disease activity scores, where available. We included 213 practices with 945,160 patients. Overall, we found visit counts decreased by 10.9\% (from 1,302,455 to 1,161,051) between March and August 2020 compared to 2019; this drop was most dramatic during the month of April (-22.3\%). Telemedicine visits increased from $0 \%$ to a mean of $12.1 \%$. Practices in SIP states had more dramatic decreases in visits, (11.5\% vs. $5.3 \%)$. We found no major differences in primary diagnoses or disease activity across the two periods. We detected a meaningful decrease in rheumatology visits in March-August 2020 during the SARS-CoV-2 global pandemic compared to the year prior with a concomitant increase in the use of telemedicine. Future work should address possible adverse consequences to patient outcomes due to decreased contact with clinicians.
\end{abstract}

Keywords COVID-19 $\cdot$ Patient registry $\cdot$ Health services

Gabriela Schmajuk

gabriela.schmajuk@ucsf.edu

1 Division of Rheumatology, Department of Medicine, University of California, 4150 Clement St 111R, San Francisco, CA 94121, USA

2 Seattle Children's Hospital, Seattle, WA, USA

3 University of Alabama at Birmingham, Birmingham, AL, USA

4 University of Nebraska Medical Center, Omaha, NE, USA

5 The National Databank for Rheumatic Diseases, Wichita, KS, USA

6 Practice, Advocacy \& Quality, American College of Rheumatology, Atlanta, GA, USA

7 San Francisco Veterans Affairs Medical Center, San Francisco, CA, USA

\section{Introduction}

The SARS-CoV-2 global pandemic caused major disruptions to medical care in the U.S. Pandemic-associated precautions, such as shelter-in-place (SIP) orders, associated closures of many ambulatory care offices, and the widespread cancelation of elective procedures in anticipation of surges in inpatient admissions resulted in dramatic decreases in outpatient visits. During the 2 weeks in mid-March 2020, when COVID-19 cases started to rise exponentially, outpatient visits across the U.S. declined by an estimated 40\% [1].

Several studies have described trends in outpatient care delivery and telemedicine over the course of the pandemic. For example, an analysis of commercial and Medicare Advantage insurance claims from 16 million enrollees showed dramatic decreases in in-person visits, increases in telemedicine visits, and modest drops in overall visits between January and June 2020 (telemedicine and in-person 
visits combined dropping from 103.5 to 94.1 per 1000 enrollees, a $-9.1 \%$ change) [2]. One study examined disruptions in rheumatology care using data from the Arthritis and Rheumatology Associates (AARA) network, which represents approximately 300 full time practicing rheumatology clinicians across 27 states, found a $24 \%$ decrease in visits early in the pandemic and exponential growth of telemedicine visits [3].

In this study, we used the American College of Rheumatology's RISE registry to examine the relationship between pandemic and pre-pandemic visit counts and telemedicine utilization; whether these were affected by state-level shelter-in-place (SIP) orders; and whether patients with faceto-face visits during the pandemic were more likely to be sicker, as measured by disease activity in patients with rheumatoid arthritis (RA).

\section{Methods}

\section{Data source}

Data were derived from the American College of Rheumatology (ACR)'s Rheumatology Informatics System Effectiveness (RISE) registry. RISE is a national, electronichealth-record (EHR)-enabled registry that passively collects data on all patients seen by participating practices, reducing the selection bias that may be present in more highly selected data sources. As of August 2020, RISE held data from 1214 providers in 233 practices from 40 U.S. states, representing approximately $30 \%$ of the U.S. clinical rheumatology workforce. Available data included patient demographics, including 9-digit ZIP code; diagnoses; medications; laboratory results; disease activity scores; and encounter type and dates. Information about RISE practices was also provided.

\section{Study population}

Patients included in this study were from practices that participated in RISE from January 2019 through August 2020 (inclusive) and had visit data available throughout $(N=213)$. To account for the seasonal variation, we compared data from during the pandemic (March-August 2020) to data from the same time period in 2019 (March-August 2019).

\section{Outcomes}

\section{Outpatient rheumatology visits}

The main outcome in this study was overall visit count at the practice level during each time period (pandemic vs. pre-pandemic) defined as any encounter with a healthcare provider in the RISE practice, exclusive of drug trial visits.
We calculated the total number of visits and the number of telemedicine visits for each practice. We then calculated the percentage change in the visit count (for total visits and for telemedicine visits) in 2020 compared to 2019 . Telemedicine visits were identified by current procedural terminology (CPT) code $(99,441,99,442,99,443,98,966$, 98,967 and 98,968 ) and place of service (POS)/modifiers (GQ, GT, 95 and POS 02) [4]. Initial encounters were identified if a visit was the first visit a patient had recorded within the RISE registry; otherwise, encounters were considered to be follow-up visits.

\section{Primary rheumatic diagnoses}

We identified primary rheumatic diagnoses based on having at least 2 ICD codes at least 30 days apart during 2019 or 2020 and categorized according to the following codes: systemic lupus erythematosus [SLE; 710.0, 710.00 or M32x (except M32.0)], rheumatoid arthritis (RA) [(714.x, M05x or M06x (except 714.89, 714.9 and M06.4)], psoriatic arthritis (696.0 and L40.50), other spondyloarthritis (711.x, 713.x, 720.x, 99.3, M02.30 or M46.90), other inflammatory arthritis (714.89, 714.9 or M06.4), ANCAassociated vasculitis (446.4, 446.7, 447.6, M30.0, M31.30 or M31.7), giant cell arteritis (446.5 or M31.6), other vasculitis (446.1, 709.1, M30.3 or L95.8), scleroderma (710.1, M34.0, M34.1, M34.8X, or M34.9), Sjogren's (710.2 or M35.0), mixed connective tissue disease (710.8 or M35.1), polymyalgia rheumatica (725.x or M35.3), myositis $(710.3,710.4, \mathrm{G} 72.41, \mathrm{G} 72.49$ or M33.x), and sarcoidosis (135.x, D86.0 or D86.9). If patients met criteria for more than one condition, we applied a hierarchy based on one used in previously published work [5]. For patients with multiple diagnoses that did not fit into a simple hierarchy (e.g., rheumatoid arthritis and vasculitis) patients were categorized as having "more than 1" rheumatic diagnosis. Patients not falling into one of these categories were included in the "Other" group (including patients who may have had just 1 of any of the above codes). Patients with none of these conditions with at least 2 ICD codes at least 30 days apart for osteoarthritis (715.00, 715.1, or M15-M19) were categorized as "osteoarthritis only."

\section{RA disease activity}

Clinical Disease Activity Index (CDAI; range 0-76) and Routine Assessment of Patient Index Data 3 (RAPID3; range $0-30)$ scores were extracted where available. If there was more than 1 score available during the time period, the most recent score was used in the analysis. 


\section{Other predictors and covariates}

\section{Demographics}

Patient characteristics included age, sex, race/ethnicity, insurance, national Area Deprivation Index (ADI; an area-level measure of socioeconomic status (SES) based on patient 9-digit ZIP code, with a range of 1-100, with higher scores reflecting more deprivation, and thus, lower SES) [6].

\section{Comorbid conditions}

Charlson comorbidity index score was calculated according to the Deyo modification and included the patient's rheumatic diagnosis [7].

\section{Travel time}

We calculated each patient's travel time to their practice, since we hypothesized this could affect healthcare service utilization. This was calculated using the 9-digit ZIP codes of the patient's residence and their practice location: 9-digit zip codes were converted to latitude and longitude coordinates with SAS ZIP + 4 geocoding lookup table [8]. For each pair of patient-practice coordinates, the URL Access Method within the SAS software was used to access the Google Map API and extract the approximate driving time each time the site is accessed. Patients missing 9-digit ZIP codes or who had no matched ZIP codes in the SAS lookup table were excluded from the multi-variate analysis $(N=104,564 ; 11 \%$ of the cohort). For patients who had no 9-digit ZIP in the lookup table but had same 5 -digit zip as their practice $(N=16,381)$, we assigned them a travel time equal to the 25 th percentile travel time for other patients attending the same practice. Median practice times were then re-calculated with these patients included.

\section{Practice characteristics}

Practice characteristics included practice type (single specialty, solo practitioner, multi-specialty, health system and other); practice size (number of providers; number of eligible patients in each practice during study period); EHR vendor types; U.S. geographic division; and number of years contributing data to RISE.

\section{Regional shelter-in-place orders}

We obtained publicly available state-specific information on shelter-in-place (SIP) orders related to the pandemic and categorized each practice as being in a state where SIP was instituted in March 2020 vs. later or not at all [9].

\section{Statistical analysis}

Descriptive statistics were used to examine patient and practice characteristics during the pandemic and pre-pandemic periods. We used $t$-tests to compare changes in overall visit counts and telemedicine visit counts during each time period, overall and by month. We also used $t$-tests to compare the proportion of visits that were identified as initial visits during each period.

To further assess the association between the SIP status and practice-level changes in visit count, we used multi-level linear regression to assess differences in visit counts each month from March through August 2020 compared to March through August 2019; we included the following covariates: practice type, patient count, median travel time, geographic division, and time period (pre-pandemic vs. pandemic).

We also compared mean disease activity scores for RA patients across the two periods. Analyses were performed using Stata 16 (StataCorp. 2017. College Station, TX: StataCorp LLC).

\section{Results}

Among the 213 practices included, 120 (56.3\%) were single-specialty groups, $32.9 \%$ were solo practices, and $9.9 \%$ multi-specialty groups. The median number of patients per rheumatology practice was 3239 (IQR 1678-5628). Practices were most commonly in the South Atlantic and Pacific geographic divisions, and the majority of practices were in states with shelter-in-place orders $(86.4 \%)$. The median travel time at the practice level was 23 min (IQR 20-27). Other practice characteristics are listed in Table 1.

There were 945,160 unique patients included in this study. 774,109 of them had at least 1 visit pre-pandemic and 664,658 had at least 1 visit during the pandemic. Sociodemographic characteristics of patients were similar during the two periods: $75 \%$ of the patients were female, with a mean (SD) age of 62 (15) years. Primary diagnoses were also similar during the two periods: although most patients fell into the "other" category (48.9\%), the most common diagnosis was rheumatoid arthritis, followed by osteoarthritis, other inflammatory arthritis, and systemic lupus erythematosus (SLE). Patient median travel time to their corresponding practice was 23 min (IQR 17-36). The mean Charlson comorbidity index score was $0.9 \pm 1.2$ (see Table 2).

Overall, visit counts dropped $10.9 \%$ during the pandemic compared to pre-pandemic $(1,302,455-1,161,051)$. The decrease was most dramatic in April 2020 (- 22.3\%, $p<0.05$; Fig. 1). As total visits decreased starting in March 
Table 1 Characteristics of practices during the SARS-CoV-2 pandemic (March-August 2020) and the same period a year prior (March-August 2019), $N=213$

\begin{tabular}{|c|c|c|}
\hline & $N$ & $\%$ \\
\hline \multicolumn{3}{|l|}{ Practice type } \\
\hline Single specialty group practice & 120 & 56.3 \\
\hline Solo practitioner & 70 & 32.9 \\
\hline Multi-specialty group practice & 21 & 9.9 \\
\hline Health system & 2 & 0.9 \\
\hline \multicolumn{3}{|l|}{ Number of providers per practice } \\
\hline Median (IQR) & $3(1-6)$ & \\
\hline \multicolumn{3}{|l|}{$\begin{array}{l}\text { Number of eligible patients in each } \\
\text { practice }\end{array}$} \\
\hline Median (IQR) & $3239(1678-5628)$ & \\
\hline \multicolumn{3}{|c|}{$\begin{array}{l}\text { Median drive time of the patients within } \\
\text { each practice, min }\end{array}$} \\
\hline Median (IQR) & $23(20-27)$ & \\
\hline \multicolumn{3}{|l|}{ EHR system } \\
\hline NextGen & 88 & 41.3 \\
\hline eClinicalWorks & 30 & 14.1 \\
\hline eMDs & 21 & 9.9 \\
\hline Amazing charts & 17 & 8.0 \\
\hline Other & 53 & 26.8 \\
\hline \multicolumn{3}{|l|}{ Years contributing data to RISE } \\
\hline Median (IQR) & $4(3-5)$ & \\
\hline Located in a shelter-in-place state & 184 & 86.4 \\
\hline \multicolumn{3}{|l|}{ Geographic division } \\
\hline New England & 6 & 2.8 \\
\hline Mid-Atlantic & 26 & 12.2 \\
\hline East North Central & 22 & 10.3 \\
\hline West North Central & 10 & 4.7 \\
\hline South Atlantic & 56 & 26.3 \\
\hline East South Central & 20 & 9.4 \\
\hline West South Central & 26 & 12.2 \\
\hline Mountain & 10 & 4.7 \\
\hline Pacific & 37 & 17.4 \\
\hline
\end{tabular}

2020, the proportion of telemedicine visits increased, with a peak in April 2020 at $21.6 \%$ of total visits vs. $0 \%$ in 2019. Over the 6-month period in 2020 , telemedicine visits accounted for $12.1 \%$ of total visits. Initial visits accounted for $9.4 \%$ of visits in pre-pandemic and $7.5 \%$ during the pandemic $(p<0.001)$.

Practices in SIP states had greater decreases in visits during the pandemic compared to practices not in SIP states (11.5 vs. 5.3\% over the 6-month period; see Fig. 2). This relationship remained not significant even after adjusting for practice type, patient count, median travel time, geographic division and time period ( 12.5 vs. $4.8 \%, p<0.05)$.

We observed small, but not clinically meaningful differences in CDAI scores for RA patients: in 2020, mean CDAI score was $10.1 \pm 9.6$ vs. $9.5 \pm 9.6$ in 2019. Mean RAPID3 did not change $10.0 \pm 6.7$ in 2020 vs. $10.1 \pm 6.8$ in 2019 . However, we did find that proportion of RA patients with documented disease activity scores was significantly lower during the pandemic period-decreasing from $41 \%$ in 2019 to just $27 \%$ in $2020(p<0.01)$.

\section{Discussion}

Using RISE data from 2019 and 2020, we found significant decreases in rheumatology outpatient visits during the SARS-CoV-2 pandemic compared to the year before. The decrease was most dramatic in April and May 2020, although visits rebounded to near pre-pandemic levels by June 2020 and dropped again during the summer months. On the other hand, telemedicine use increased dramatically, accounting for $0 \%$ of visits pre-pandemic to $12.1 \%$ of visits during March through August 2020. Practices in SIP states saw greater decreases in total visits than those in non-SIP states, although practices in non-SIP states still dropped by $5.4 \%$. It seems likely that a meaningful fraction of patients, many of whom are immunosuppressed, may have altered their behavior and reduced their healthcare utilization regardless of local government recommendations. There may also be a population of deferred consultations with rheumatologists, as we observed a significant decrease in initial visits. We did not find that patients with RA with disease activity assessments had higher disease activity during the pandemic compared to pre-pandemic, although our sample size was limited.

The decreases in total visit counts observed in rheumatology practices in this data source are consistent with those reported using commercial and Medicare Advantage claims during a similar period: $-10.9 \%$ in RISE vs. $-9.1 \%$ in Optum/Medicine Advantage. Our findings are also consistent with the study using data from the Arthritis and Rheumatology Associates (AARA) network, which found similar decreases in overall visits and dramatic increases in the use of telemedicine [iii], as well as other studies of subspeciality care [10]. Telemedicine use is widely reported since the pandemic began in rheumatology practices and is speculated to represent a paradigm shift in the provision of outpatient care, as such virtual visits have been shown to be acceptable for both rheumatology clinicians and their patients [11-15]. The use of telemedicine presents several unique challenges, including how to assess outcomes in patients with inflammatory arthritis without the ability to perform a physical exam. On the other hand, this change presents opportunities for innovation in the care of patients with rheumatic diseases, including, but not limited to, the use of digital health applications to monitor patient symptoms and patient-reported outcomes remotely on a routine basis [16]. 
Table 2 Characteristics of patients during the SARSCoV-2 pandemic (MarchAugust 2020) compared to the same period a year prior (March-August 2019)

\begin{tabular}{|c|c|c|c|c|}
\hline & \multicolumn{2}{|c|}{$\begin{array}{l}\text { Patients with visits during } \\
\text { March through August } 2019 \\
N=774,109\end{array}$} & \multicolumn{2}{|c|}{$\begin{array}{l}\text { Patients with visits } \\
\text { during March through } \\
\text { August } 2020 \\
N=664,658\end{array}$} \\
\hline & $N$ & $\%$ & $N$ & $\%$ \\
\hline Female & 578,210 & 74.7 & 496,415 & 74.7 \\
\hline Age, mean (SD) & $62.0(15.4)$ & & $61.5(15.3)$ & \\
\hline ADI, median (interquartile range) & $40(20-64)$ & & $40(21-64)$ & \\
\hline Residing in a shelter-in-place state & 687,462 & 88.8 & 587,464 & 88.4 \\
\hline Drive time to rheumatology practice, $\min$ & $n=689,149$ & & $n=593,463$ & \\
\hline Median, (IQR) & $23(17-36)$ & & $23(17-36)$ & \\
\hline Initial visits out of total visits, $\%$ & 9.4 & & 7.5 & \\
\hline \multicolumn{5}{|l|}{ Race/ethnicity } \\
\hline White & 516,382 & 66.7 & 436,164 & 65.6 \\
\hline Hispanic & 47,755 & 6.2 & 39,057 & 5.9 \\
\hline African American & 51,210 & 6.6 & 43,856 & 6.6 \\
\hline Asian & 15,383 & 2.0 & 12,162 & 1.8 \\
\hline Other/Mixed & 3208 & 0.4 & 2754 & 0.4 \\
\hline Unknown/declined & 140,171 & 18.1 & 130,665 & 19.7 \\
\hline \multicolumn{5}{|l|}{ Insurance } \\
\hline Private & 252,073 & 32.6 & 226,966 & 34.2 \\
\hline Medicare & 238,797 & 30.9 & 207,226 & 31.2 \\
\hline Any medicaid & 18,754 & 2.4 & 17,286 & 2.6 \\
\hline Other & 30,900 & 4.0 & 25,487 & 3.8 \\
\hline Unknown & 233,585 & 30.1 & 187,693 & 28.2 \\
\hline \multicolumn{5}{|l|}{ Diagnosis } \\
\hline Rheumatoid arthritis & 186,020 & 24.0 & 167,731 & 25.2 \\
\hline Osteoarthritis only & 123,643 & 16.0 & 98,037 & 14.8 \\
\hline Other inflammatory arthritis ${ }^{\mathrm{a}}$ & 60,116 & 7.8 & 53,124 & 8.0 \\
\hline SLE & 33,942 & 4.4 & 30,161 & 4.5 \\
\hline Polymyalgia rheumatica & 13,506 & 1.7 & 11,292 & 1.7 \\
\hline Mixed connective tissue disease & 5262 & 0.7 & 4667 & 0.7 \\
\hline Scleroderma & 6667 & 0.9 & 5616 & 0.8 \\
\hline Vasculitis $^{\mathrm{b}}$ & 4855 & 0.6 & 4260 & 0.6 \\
\hline Sjogren's & 1980 & 0.3 & 1572 & 0.2 \\
\hline Myositis & 4355 & 0.6 & 3887 & 0.6 \\
\hline $\begin{array}{l}\text { More than } 1 \text { rheumatic condition (excluding } \\
\text { osteoarthritis) }\end{array}$ & 1360 & 0.2 & 1243 & 0.2 \\
\hline Other $^{c}$ & 332,403 & 42.8 & 283,068 & 42.7 \\
\hline \multicolumn{5}{|l|}{ Other clinical variables } \\
\hline Charlson comorbidity index, mean (SD) & $0.9(1.2)$ & & $0.9(1.2)$ & \\
\hline RAPID3 score among RA patients & $n=190,209$ & & $n=123,213$ & \\
\hline Mean (SD) & $10.1(6.8)$ & & $10.0(6.7)$ & \\
\hline CDAI score among RA patients & $n=77,688$ & & $n=46,531$ & \\
\hline Mean (SD) & $9.5(9.6)$ & & $10.1(9.6)$ & \\
\hline
\end{tabular}

${ }^{\mathrm{a}}$ Other inflammatory arthritis included: inflammatory arthritis, psoriatic arthritis, and spondyloarthritis.

${ }^{b}$ Vasculitis included: ANCA-associated vasculitis, giant cell arteritis and other vasculitis

${ }^{\mathrm{c}}$ Other included: all patients not falling into any of the above groups.
Our study has some important limitations to note: RISE registry practices are mostly solo and single-specialty community practices, so findings may not apply to large or academic practices. We did not account for changes in the number of clinicians at each practice, which could correlate with the number of visits in a practice. Less than $50 \%$ 


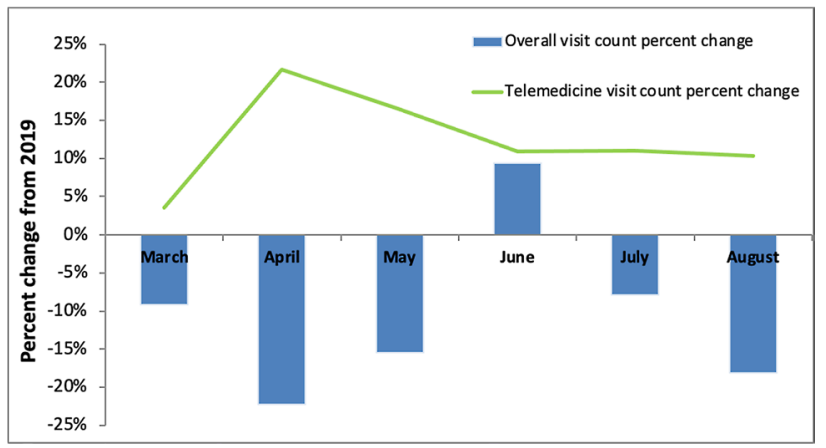

Fig. 1 Percent change in overall and telemedicine visit counts in 2020 compared to 2019 , stratified by month

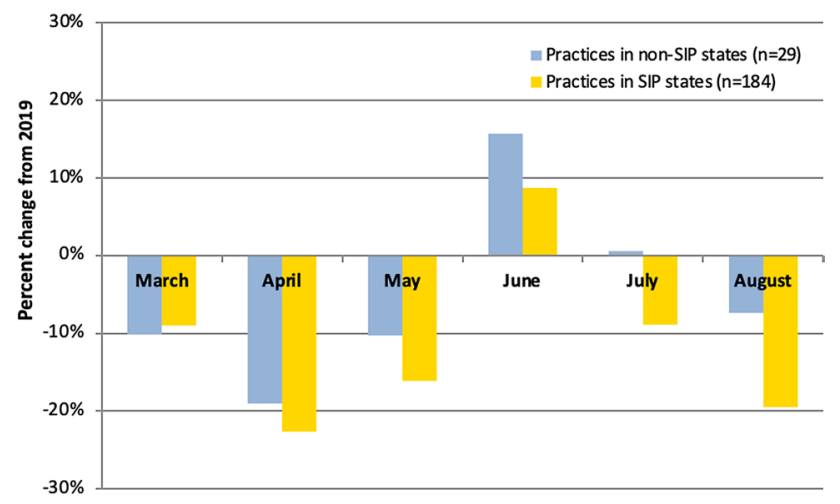

Fig. 2 Percent change in overall visit counts in 2020 compared to 2019, stratified by whether a practice was located in a shelter-in-place (SIP) state

of patients with RA had a disease activity score recorded during the study period, so our findings that disease activity did not change among RA patients with visits may be biased.

The data presented here have important implications for future research and policy work: given the dramatic decrease in visits during 2020, research using large observational datasets such as RISE will need to take this secular event into account. For example, studies that assess healthcare utilization will need to treat the pandemic period separately from time periods before the pandemic, as overall utilization will have decreased during this time, perhaps disproportionately across different patient characteristics such as age, SES, and drive time to practices [3]. In addition, the advent of telemedicine may affect the collection of disease activity scores and other metrics that require in-person assessments: we noted significant decreases in the proportion of RA patients with disease activity measures documented. Pay-for-performance programs that rely on collection of these measures may need to be adjusted if such changes are long-lasting.
It is still unclear whether a decreased in visits or deferred consultation with rheumatologists during the early part of the SARS-CoV-2 pandemic may have affected patient outcomes due delays in diagnosis, less frequent adjustments to medication regimens, or other decreases in healthcare utilization, which in turn could lead to higher disease activity, or an increase in medication safety issues. These effects could take many months (or longer) to detect. Reassuringly, studies of patients with diabetes have shown that although ambulatory care visits for this condition decreased during the pandemic, medication refills and outcomes such as the hemoglobin A1C level were not affected [17]. Future work should address possible changes in patient outcomes resulting from decreased contact with rheumatology clinicians during the pandemic.

Author contributions GS contributed to the study conception and design. Material preparation, data collection and analysis were performed by JL, GS and TJ. All the authors contributed to data interpretation. The first draft of the manuscript was written by GS, and all the authors commented on subsequent versions of the manuscript. All the authors read and approved the final manuscript.

Funding Drs. Yazdany and Schmajuk are supported by the Russell/ Engleman Medical Research Center for Arthritis. Data collection was supported by the American College of Rheumatology (ACR)'s RISE Registry. However, the views expressed represent those of the authors, not necessarily those of the ACR.

Availability of data and material The American College of Rheumatology (ACR) owns the data in the RISE registry, and UCSF, as a Data Analytic Center for the ACR, has access to the data for specific research projects, including this one, but is contractually obligated through data use agreements with practices to not share this data, even in a deidentified state.

Code availability The code used to analyze RISE data will be made available upon request.

\section{Declarations}

Conflict of interest Dr. Ringold is supported by the Childhood Arthritis and Rheumatology Research Alliance and receives research support from BMS. Dr. Curtis receives grants and personal fees from Abbvie, Amgen, BMS, Corrona, Eli Lilly, Jannsen, Myriad, Pfizer, Reneron, Radius, Rochen, and UCB outside of the submitted work. Ms. Li, Drs. Yazdany, Michaud, Yun and Schmajuk, and Ms. Johansson have no conflicts of interest.

Ethics approval This study was approved by the Western IRB (WIRB Work Order \#1-797516-1) and UCSF Committee on Human Research (17-24073).

Consent to participate Waived by IRB.

Consent for publication Waived by IRB. 


\section{References}

1. Ateev M, Michael C, David L, Hilary H, David C (2020) The impact of the COVID-19 pandemic on outpatient visits: practices are adapting to the new normal. The Commonwealth Fund. https://www.commonwealthfund.org/publications/2020/jun/ impact-covid-19-pandemic-outpatient-visits-practices-adaptingnew-normal. Accessed 1 Dec 2020

2. Patel SY, Mehrotra A, Huskamp HA, Uscher-Pines L, Ganguli I, Barnett ML (2021) Trends in outpatient care delivery and telemedicine during the COVID-19 pandemic in the US. JAMA Intern Med 181(3):388-391. https://doi.org/10.1001/jamaintern med.2020.5928

3. George MD, Danila MI, Watrous D, Reddy S, Alper J, Xie F, Nowell WB, Kallich J, Clinton C, Saag KG, Curtis JR (2021) Disruptions in rheumatology care and the rise of telehealth in response to the COVID-19 pandemic in a community practicebased network. Arthritis Care Res (Hoboken). https://doi.org/10. 1002/acr.24626.10.1002/acr.24626

4. Jedrek W, Marat F, Blake C et al (2020) Telehealth transformation: COVID-19 and the rise of virtual care. J of the Am Med Inform Association 27(6):957-962. https://doi.org/10.1093/jamia/ocaa0 67

5. Patterson S, Schmajuk G, Evans M et al (2019) Gaps in ambulatory patient safety for immunosuppressive specialty medications. Jt Comm J Qual Patient Saf 45(5):348-357. https://doi.org/10. 1016/j.jcjq.2018.12.003

6. University of Wisconsin-Madison (2018) Neighborhood Atlas. Department of Medicine, University of Wisconsin School of Medicine and Public Health. https://www.neighborhoodatlas.medicine. wisc.edu/. Accessed 1 Dec 2020

7. Deyo RA, Cherkin DC, Ciol MA (1992) Adapting a clinical comorbidity index for use with ICD-9-CM administrative databases. J Clin Epidemiol 45(6):613-619. https://doi.org/10.1016/ 0895-4356(92)90133-8

8. SAS Institute (2013) Geocoding data. SAS Institute. https://suppo rt.sas.com/rnd/datavisualization/mapsonline/html/geocode.html\# zip4. Accessed 21 April 2020

9. Amanda M, Christine H, Michael A, et al (2020) Timing of state and territorial COVID-19 stay-at-home orders and changes in population movement-United States, March 1- May 31, 2020. Center for Disease Control and Prevention. https://www.cdc.gov/ mmwr/volumes/69/wr/mm6935a2.htm. Accessed 1 Dec 2020
10. Anderson TS, O'Donoghue AL, Dechen T, Herzig SJ, Stevens JP (2021) Trends in telehealth and in-person transitional care management visits during the COVID-19 pandemic. J Am Geriatr Soc. https://doi.org/10.1111/jgs.17329

11. Bateman J, Cleaton N (2021) Managing patients using telerheumatology: Lessons from a pandemic. Best Pract Res Clin Rheumatol 35(1):101662. https://doi.org/10.1016/j.berh.2021.101662

12. Muehlensiepen F, Knitza J, Marquardt W, Engler J, Hueber A, Welcker M (2021) Acceptance of telerheumatology by rheumatologists and general practitioners in Germany: nationwide crosssectional survey study. J Med Internet Res 23(3):e23742. https:// doi.org/10.2196/23742

13. Matsumoto RA, England BR, Mastarone G, Richards JS, Chang E, Wood PR, Barton JL (2020) Rheumatology clinicians' perceptions of telerheumatology within the veterans health administration: a national survey study. Mil Med 185(11-12):e2082-e2087. https:// doi.org/10.1093/milmed/usaa203

14. Kavadichanda C, Shah S, Daber A, Bairwa D, Mathew A, Dunga S, Das AC, Gopal A, Ravi K, Kar SS, Negi VS (2021) Tele-rheumatology for overcoming socioeconomic barriers to healthcare in resource constrained settings: lessons from COVID-19 pandemic. Rheumatology (Oxford) 60(7):3369-3379. https://doi.org/ 10.1093/rheumatology/keaa791

15. Bos WH, van Tubergen A, Vonkeman HE (2021) Telemedicine for patients with rheumatic and musculoskeletal diseases during the COVID-19 pandemic; a positive experience in the Netherlands. Rheumatol Int 41(3):565-573. https://doi.org/10.1007/ s00296-020-04771-6

16. Kernder A, Morf H, Klemm P, Vossen D, Haase I, Mucke J, Meyer M, Kleyer A, Sewerin P, Bendzuck G, Eis S, Knitza J, Krusche M (2021) Digital rheumatology in the era of COVID-19: results of a national patient and physician survey. RMD Open 7(1):e001548. https://doi.org/10.1136/rmdopen-2020-001548

17. Patel SY, McCoy RG, Barnett ML, Shah ND, Mehrotra A (2021) Diabetes care and glycemic control during the COVID-19 pandemic in the United States. JAMA Intern Med. https://doi.org/10. 1001/jamainternmed.2021.3047

Publisher's Note Springer Nature remains neutral with regard to jurisdictional claims in published maps and institutional affiliations. 